\title{
Photonometers for coating and sputtering machines
}

\author{
P. Oupický, D. Jareš, J. Václavík, D. Vápenka \\ Institute of Plasma Physics ASCR v.v.i., TOPTEC, Sobotecká 1660, 51101 Turnov, Czech Republic
}

\begin{abstract}
The concept of photonometers (alternative name of optical monitor of a vacuum deposition process) for coating and sputtering machines is based on photonometers produced by companies like SATIS or HV Dresden. Photometers were developed in the TOPTEC centre and its predecessor VOD (Optical Development Workshop of Institut of Plasma Physics AS CR) for more than 10 years. The article describes current status of the technology and ideas which will be incorporated in next development steps. Hardware and software used on coating machines B63D, VNA600 and sputtering machine UPM810 is presented.
\end{abstract}

\section{Optical conception of photonometers}

Photonometers generally consist of illuminating system, optical bench with vacuum windows and ports, a holder for test sample, holders for exchangeable filters, adjustable holders for photosensors, photosensors and a measuring unit with control software.

A concept of reflex-transmission photonometer with perpendicular throughput of light via a test sample was used for coating machines B63D and VNA600. Reflectance signal is obtained with use of a beam-splitter as well as reference signal for monitoring intensity of the light source. There is possible to use alternatively the photonometer with a small angle of incident light on test sample without need for beam-splitter.

A suitable arrangement for the sputtering machine UPM810 is a transmission photonometer with a light beam passing directly via test sample hold on rotation device together with sputtered substrates.

\section{Illuminating systems}

There are two possible illuminating systems - with continuous light beam or with chopped light beam.

The illuminating system with continuous light sources is suitable for machines where the interruption of light is provided by movement of the test sample itself i.e. in sputtering machine UPM810. There is possible to use halogen lamp or LED diodes with collimating optics.

The illuminating system with chopped light is suitable for all other type of coating machines. Halogen lamp with a mechanical chopper or a LED driven in pulsed mode by its control electronics can be used.

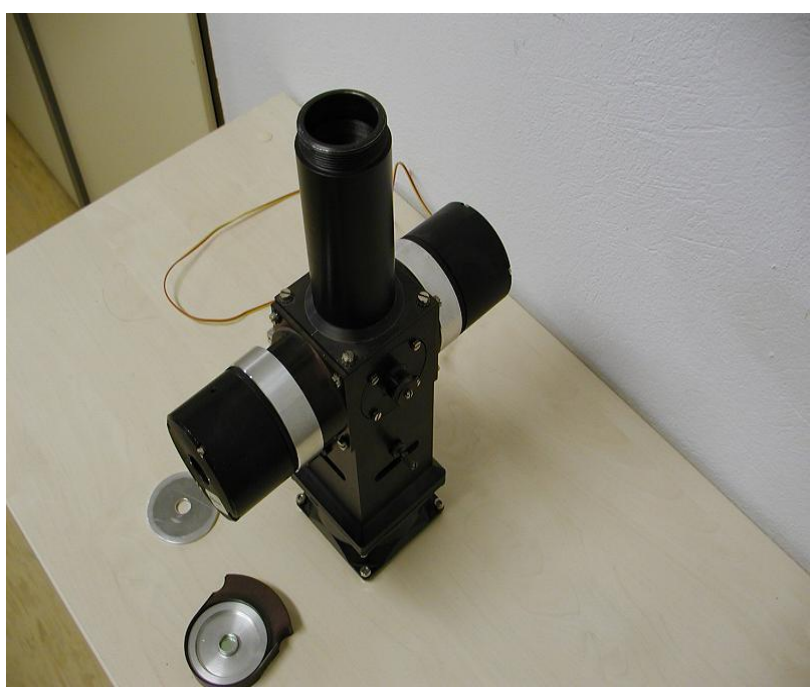

Fig. 1. Body of a photonometer used on coating machine B63D

\section{Optical path of photonometers}

The illumination system consist of optical stop on top of a halogen lamp or LED diode, alternatively mechanical chopper, beam-splitter which divides light for reference channel as well as deflect light reflected from sample. Transmitted light from beam-splitter is collimated with the lens and then continues to a test sample and here is reflected in the same path or transmitted to collimator in front of transmission photosensor with transmission filter. The photosensor and filter for reflected beam is placed next to beam-splitter to detect amount of reflected light. The whole optical bench/path has a classical known optical schema.

This is an Open Access article distributed under the terms of the Creative Commons Attribution License 2.0, which permits unrestricted use, distribution, and reproduction in any medium, provided the original work is properly cited. 


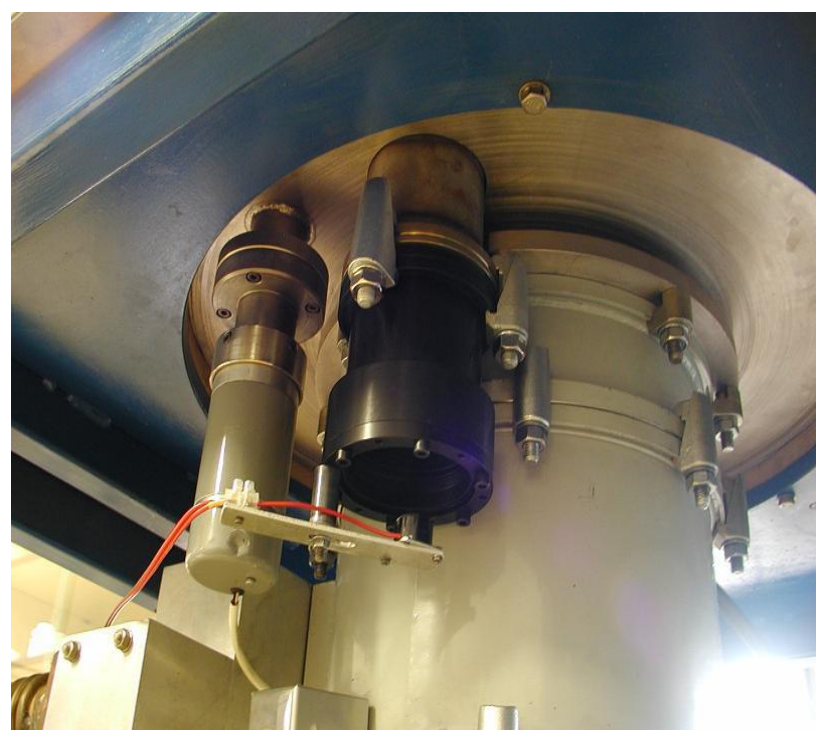

Fig. 2. Example of a vacuum port with a simplest holder for illuminating system with LED and associated optics

Second type of photonometer uses the tilted reflection/transmission from/through the test sample. The divider is substituted by a mirror which reflects the light with the tilt near to $45^{\circ}$ into the reference photosensor and the main beam is passing through a stop in the mirror and after a collimation is going to the test sample. There the beam is transmitted or reflected with small tilt from/through the test sample. The transmitted part of beam is then focused to the transmission photosensor and the reflected part returns to the first tilted mirror from which is reflected to the reflection photosensor. All other details are the same as in perpendicular system.

Transmission photonometer for sputtering machines can be constructed with a single or dual beam illuminating systems. In the case of the dual one the first branch of light is used as a reference channel and second branch is going through the sputtered test sample.

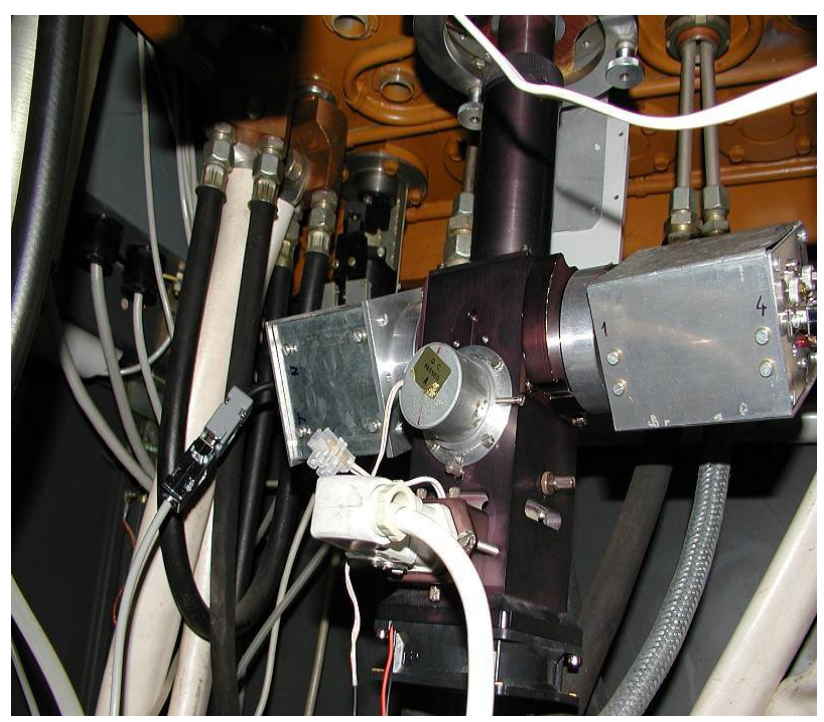

Fig. 3. Photonometer in the coating machine B63D with prototypes of photosensors

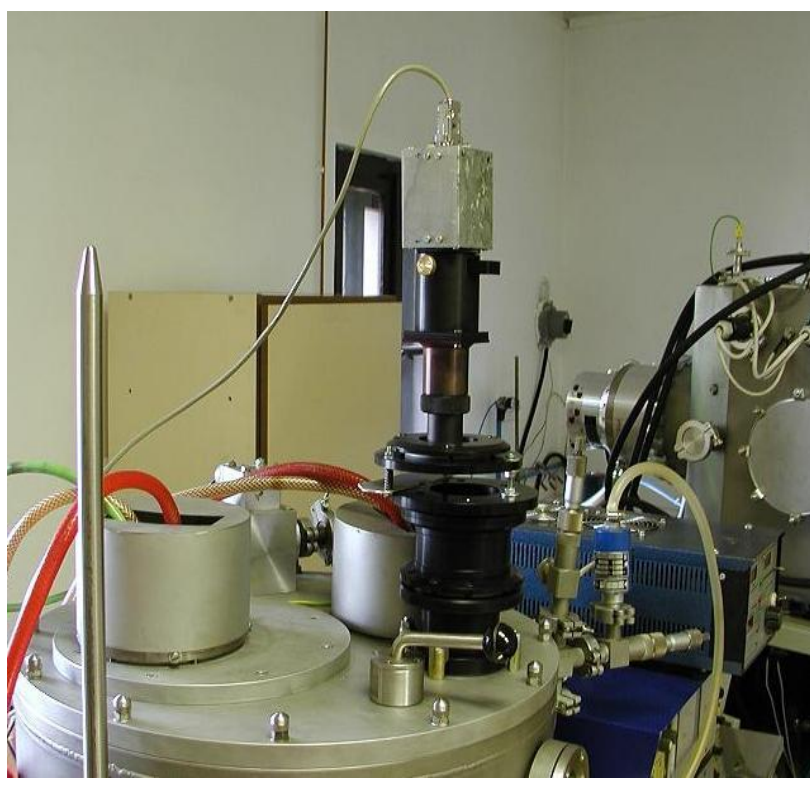

Fig. 4. Photosensor of transmitted light on the sputtering machine UPM810 with prototype of vaccum port and holder

Transmission photonometer for sputtering machines can be constructed with a single or dual beam illuminating systems. In the case of the dual one the first branch of light is used as a reference channel and second branch is going through the sputtered test sample.

\section{The concept of photosensors}

These photosensors are coupled with the detachable coupling (usually a thread) with a holder of replaceable optical filters and a focusing lens with the possibility of a fine set up in order to the centre focused beam onto sensor. This beam is reaching the photodiode coupled usually direct with an amplifier and related electronic.

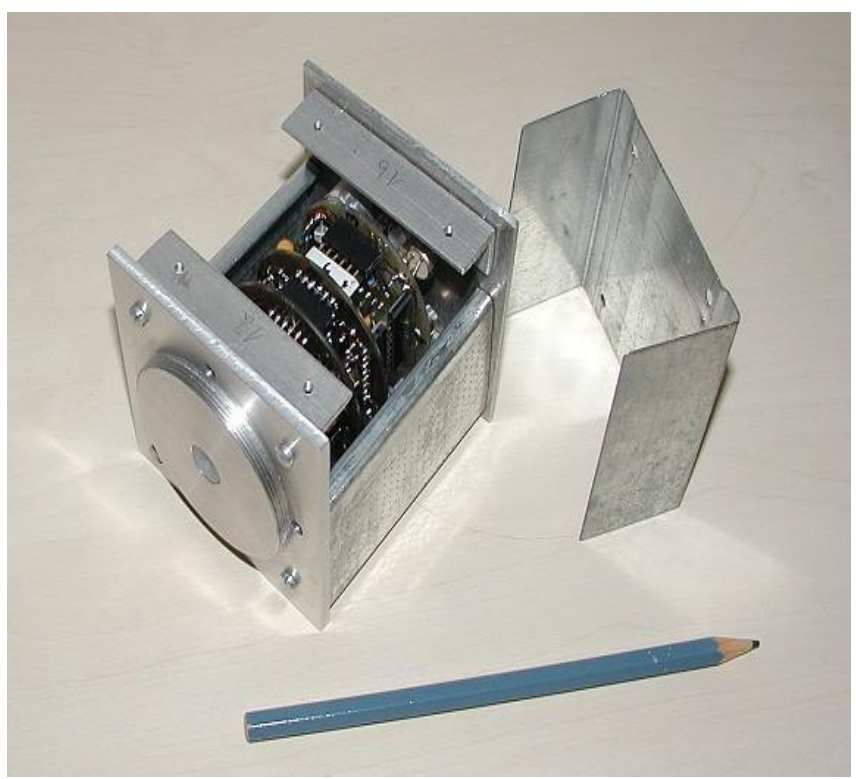

Fig. 5. Prototype of the test version of photosensor

The reference and reflection photosensors are usually closely coupled to beam-splitter of the illuminating 
system. The transmission photosensor is usually coupled with its holder including a removable filter and a focusing lens.

Photosensors can be alternatively replaced by a spectrometer coupled with lens adapter and optical fiber.

\section{Holders for photosensors}

These holders are constructed with possibility to set up position of photosensors very accurately into the centre of optical path of the transmitted or reflected light beam. The example of holder is visible in the figure 4 and 6 . This holder is possible to set up with three screws, a moveable plate and spacers.

\section{Electronics of photosensors}

Photodiode with enhanced blue sensitivity is usually used in photosensors. The photodiode is directly coupled with an amplifier on the same PCB in order to minimize noise. The amplifier can be connected via analog line directly to central unit or to the board with an $\mathrm{AD}$ converter and microcontroller. The digitized data are then transferred to the central unit via digital communication line. Central unit is realized by personal or industrial computer.

The concept of transmission, reflection and reference photosensor is almost the same and they differ only in their sensitivity.

\section{Vacuum ports}

The vacuum machines must be equipped by optical ports with a tilted windows (elliptical) produced from BK7 or a fused silica. A high transmission in UV region is favourable, when an UV LED is used as illumination source. If the photonometer is used only in VIS or NIR region then it is possible use the float glass or similar one.

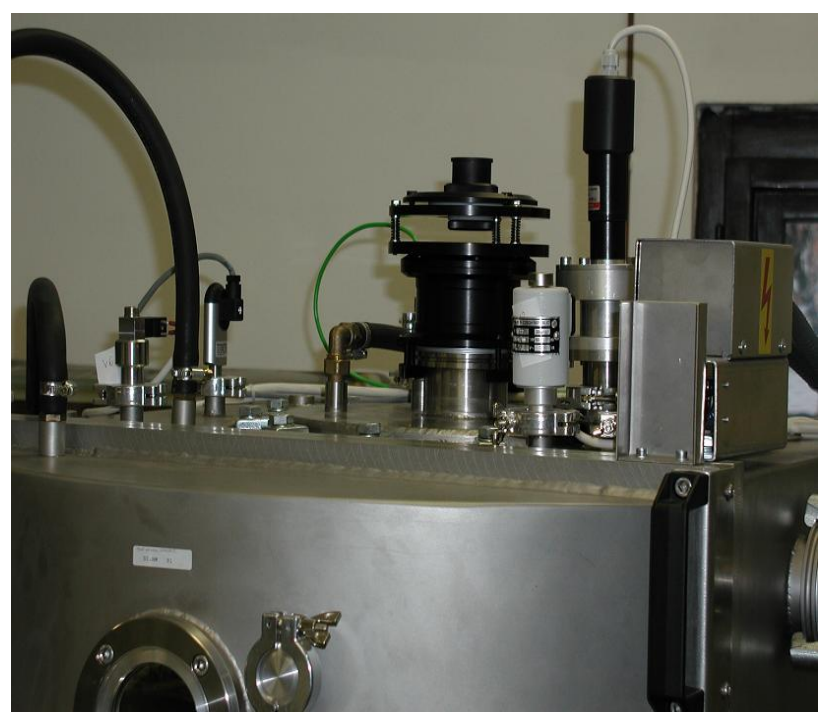

Fig. 6. Vacuum port on the top side of vacuum chamber of coating machine VNA 600 with the holder for photosensor

\section{Electronics of photometers}

Chopped source of light with frequency about 30-40 Hz is usually used for illumination. If a halogen lamp is used then the light is chopped with a mechanical rotating shutter. In case of LEDs the light is chopped with electronic system. In both systems of illuminating a stabilised DC power source is used for lamps powering. Signal from analog photosensors is fed to the central measuring unit through shielded cable. Digital ones use a RS232 or RS485 data lines for data transfer. Personal or an industrial computer can be used directly as a control unit.

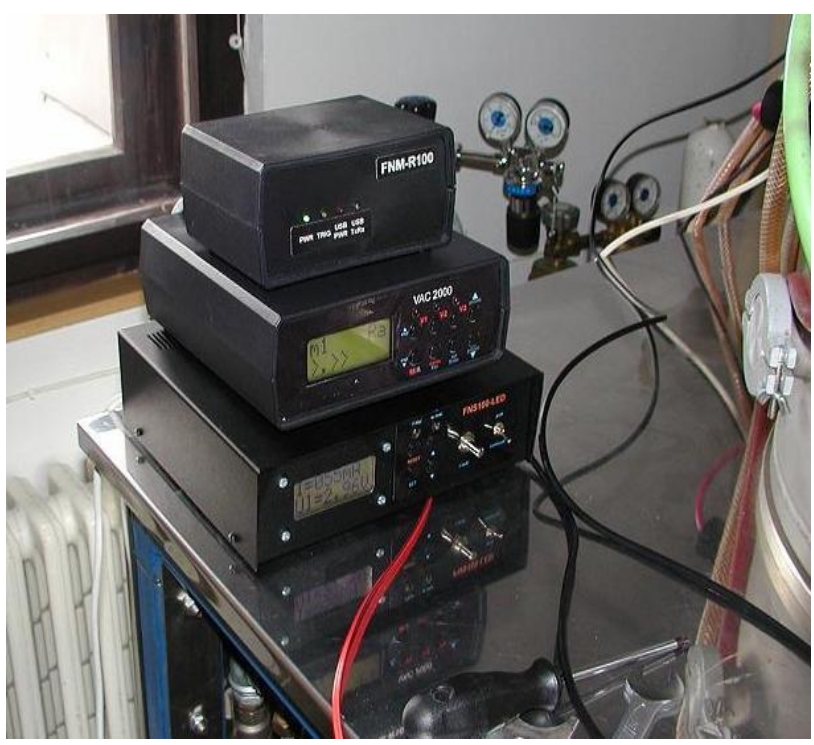

Fig. 7. A socket-outlet adaptor for RS485 FNM-R100 (up), a source for LED FNM-Z100 (down) and a vacuum measurement and controller VAC 2000 (in the middle)

\section{Software}

Photonometers are usually used as a measuring device itself or it is possible to control them via computer. In this case the computer controls functional parameters of photonometers and provides graphic visualization of measuring process, data collection and data processing. 


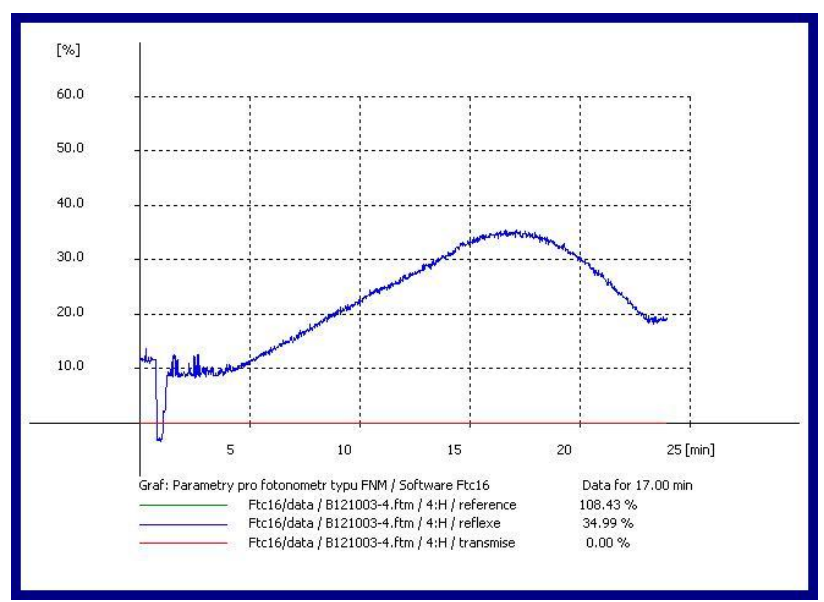

Fig.8. An example of the graphic result from coating process

\section{Conclusion}

Photonometers are used instead of quartz controllers as the main devices for control of all thin-film processes. The systems developed and produced in VOD and following TOPTEC are fully ready for the use in thinfilms processes on coating or sputtering machines.

This work was supported by the European Regional Development Fund and the Ministry of Education, Youth and Sports of the Czech Republic in the Project No. CZ.1.05/2.1.00/03.0079: Research Center for Special Optics and Optoelectronic Systems (TOPTEC).

\section{References}

1. P. Oupicky: JMO, 7-8, p. 211 (2008) 